\title{
MECHANICAL VIBRATIONS REDUCE THE INTERVERTEBRAL DISC SWELLING AND MUSCLE ATROPHY FROM BED REST
}

Nilsson Holguin ${ }^{1}$, Jesse Muir ${ }^{1}$, Harlan J. Evans ${ }^{2}$, Yi-Xian Qin ${ }^{1}$, Clinton Rubin ${ }^{1}$, Mark Wagshul ${ }^{1}$, and Stefan Judex ${ }^{1}$

${ }^{1}$ Department of Biomedical Engineering, State University of New York-Stony Brook, Stony Brook, NY11794

${ }^{2}$ Johnson Space Center, NASA, Houston, TX 77058

E-mail: Nilsson.Holguin@gmail.com

\section{INTRODUCTION}

Loss of functional weight bearing, such as experienced during space flight or bed rest (BR), distorts intervertebral disc (IVD) [1] and muscle morphology [2]. IVDs are avascular structures consisting of cells that may derive their nutrition and waste removal from the load induced fluid flow into and out of the disc [3]. A diurnal cycle is produced by forces related to weight bearing and muscular activity, and comprised of a supine and erect posture over a $24 \mathrm{hr}$ period. A diurnal cycle will include a disc volume change of $\sim 10-13 \%$. However, in space there are little or no diurnal changes because of the microgravity, which removes the gravitational load and compressive forces to the back muscles. The BR model and the etiology of the disc swelling and muscle atrophy could provide insight into those subjects confined to bed for chronic disease/injury and aging. We hypothesize that extremely low-magnitude, highfrequency mechanical vibrations will abate the disc degeneration and muscle loss associated with long-term BR.

\section{METHODS}

Eighteen normal subjects were recruited. The study, thus far, consisted of 10 males and 8 females $(33 \pm 7 \mathrm{yrs})$. Control subjects underwent BR for up to $90 \mathrm{~d}$ and were scanned by computed tomography (CT) at $0 d(n=10), 45 d(n=4)$, or $90 d(n=6)$ and MRI at $0 d(n=2), 60 d(n=2), 90 d(n=2)$ and $8 d$ after completion of BR $(n=2)$. Experimental subjects (VIBE) were subjected to 90 days of bed rest but received vibrations at $0.3 \mathrm{~g}$ and $30 \mathrm{~Hz}$ for $10 \mathrm{~min} /$ day. The VIBE subjects were scanned by CT at $0 \mathrm{~d}(\mathrm{n}=8)$ and $90 \mathrm{~d}$ $(n=8)$, and by MRI at $0 d(n=8), 60 d(n=6)$, $90 \mathrm{~d}(\mathrm{n}=7)$ and $8 \mathrm{~d}$ after completion of BR $(\mathrm{n}=7)$.

CT scans were taken between T12 and L3 to measure the IVD and back muscle volume. MRI scans were taken between T12 and S1 to measure the IVD and nucleus volume. Tissues volumes were contoured by hand. In all subjects, lean whole body mass was determined by DXA. Differences between groups were assessed by Students' T-test $(\mathrm{p}<0.05)$.

\section{RESULTS AND DISCUSSION}

Effective countermeasures to long term BR are currently not available. Exercise regimes involving walking with and without additional backpacks indicate that conventional mechanical loads applied at relatively low frequencies are unable to return disc volume to normal levels [4]. By increasing the frequency of the mechanical signal, extremely low-magnitude, high frequency whole body vibrations can be anabolic and/or anti-catabolic to muscle [5].

Data from a previous study [6] were used to compare our $90 \mathrm{~d}$ data groups to $1 \mathrm{~d}$ of BR. IVD volumes measured by CT scans (Fig.1) 
showed that after 90d of BR the volume of the IVD of control subjects increased by $55 \%$ in L1-L2 ( $>0.05)$ and $60 \%$ in L2-L3 $(p<0.05)$. In contrast, the superposition of low-level vibrations for $10 \mathrm{~min} / \mathrm{d}$ mitigated this expansion by $61 \%(\mathrm{p}<0.01)$ in $\mathrm{T} 12-\mathrm{L} 1$, $36 \%(\mathrm{p}<0.05)$ in L2-L3, and 33\% $(\mathrm{p}>0.05)$ in L1-L2. IVD volumes measured by MRI scans showed that mechanical vibrations concomitant to BR abated the IVD swelling at $60 \mathrm{~d}$ by $150 \%$ and $90 \mathrm{~d}$ by $65 \%$. Eight days after BR, the control group showed a plasticity of $9 \%$, while the VIBE group showed no residual change $(p>0.05)$. There was not any difference in the swelling of the nucleus pulposus between the control and VIBE groups.

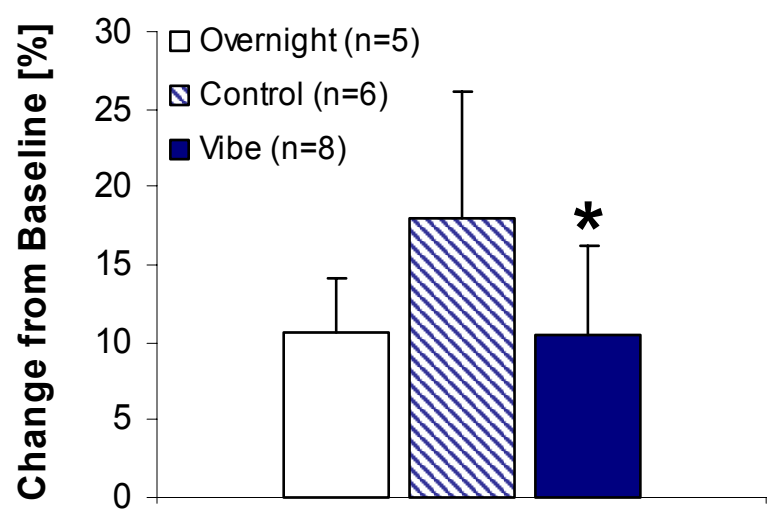

Fig. 1. IVD swelling from baseline (mean + $\mathrm{SD}$ ), as measured by CT scan in control and VIBE subjects

Mechanical vibrations reduced the back muscle atrophy from long-term BR by $33 \%$ ( $>0.05)$. Intrinsic back muscle volume was highly correlated with DXA whole body lean mass $\left(\mathrm{R}^{2}=0.97\right)$. Whole body vibrations reduced the whole body muscle mass loss from $-2.1 \%$ to $-1 \%(\mathrm{p}>0.05)$.

The MRI data showed that the differences between controls and vibrated subjects were associated with changes in the annulus fibrosus, rather than the nucleus pulposus.
The plasticity reduction posits that vibrations will not only attenuate the harmful effects of long-term BR during BR but after BR as well.

\section{SUMMARY/CONCLUSIONS}

The mechanisms by which whole body vibrations affect soft tissues are yet to be determined. However, considering the additional potential benefits of whole body vibrations on the skeletal and sensorimotor system, these mechanical signals may present a promising non-pharmacologic countermeasure of tissue degeneration both on earth as well as in space

\section{REFERENCES}

[1] A. D., LeBlanc, H. J., Evans, V. S., Schneider, R. E., Wendt, 3rd, and Hedrick, T. D. (1994). Spine, 19(7), pp. 812-817.

[2] P. E., di Prampero, and M. V., Narici, (2003). J. Biomech, 36(3), pp. 403-412.

[3] S., Holm, and A., Nachemson. (1983). Spine, 8(8), pp. 866-874.

[4] W. C., Hutton, J. A., Malko, and W. A., Fajman. (2003). Aviat Space Environ Med. 74(1), pp. 73-78.

[5] X. J. Musacchia and S. Fagette. (1997). J Gravit Physiol, 4(3), pp.49-59.

[6] J.A., Malko, W.C., Hutton, and W.A. Fajman. (2002). J Spinal Disord Tech. Apr;15 (2):157-63.

\section{ACKNOWLEDGEMENTS}

This research opportunity was funded by the National Space Biomedical Research Institute and NASA. 